\title{
Undifferentiated Pleomorphic Sarcoma of the Cheek with Surface Ulceration: Mimicking Spindle Cell Squamous Cell Carcinoma
}

\author{
Fumiko Momma*, Chihiro Onami, Takayuki Konno, Tamio Suzuki
}

Department of Dermatology, Faculty of Medicine, Yamagata University, Yamagata, Japan.

Email: *fmonma@med.id.yamagata-u.ac.jp

Received August $9^{\text {th }}, 2013$; revised September $4^{\text {th }}$, 2013; accepted September $11^{\text {th }}, 2013$

Copyright (C) 2013 Fumiko Momma et al. This is an open access article distributed under the Creative Commons Attribution License, which permits unrestricted use, distribution, and reproduction in any medium, provided the original work is properly cited.

\begin{abstract}
Undifferentiated pleomorphic sarcoma (UPS), also called malignant fibrous histiocytoma (MFH), belongs to the soft tissue tumors and is more likely in elder patients. UPS currently accounts for approximately $18 \%$ of cutaneous soft tissue sarcoma except Kaposi sarcoma. The most common sites are the extremities and head and neck. UPS is rapidly enlarging, and easily invades subcutaneous tissue and muscle without overlying skin changes. UPS tumors, which are confined to subcutaneous tissue, have been reported to be less than $10 \%$. On the other hand, the involvement of the epidermis, occasionally with ulceration, is rare. Here, we describe a case of UPS of the cheek with surface ulceration. An 83-year-old man presented with a 1-year history of a red nodule on his left cheek. The size of the nodule gradually increased. Clinical examination revealed a $55 \times 45 \mathrm{~mm}$ red, hard mass in the center of his left cheek with a large cratershaped ulcer involving the subcutaneous tissue. The bottom of the ulcer contained necrotic tissue. No cervical lymph nodes were palpable. A biopsy revealed a high-grade, pleomorphic spindle cell neoplasm and frequent abnormal mitotic figures within the dermis. We temporarily diagnosed and suspected a soft tissue sarcoma, but we could not rule out spindle cell squamous cell carcinoma (SCSCC). The patient underwent tumor resection and skin grafting. The tumor had reached the muscle layer of his left cheek with invasion and ulceration of the overlying skin. Histopathological examination of the totally resected tumor revealed a neoplasm of mesenchymal origin, rich in cells with morphology and immunohistochemical characteristics compatible with UPS. Malignant cells were not found in the epidermis. Immunohistochemical analyses were positive for vimentin, $\alpha$-anti-trypsin, $\alpha$-smooth muscle actin ( $\alpha$-SMA), and CD10, and negative for cytokeratins (CAM5.2 and AE1/AE3), lysozyme, epithelial membrane antigen (EMA), CD34, S-100, desmin, and caldesmon. Two months after total resection, the tumor recurred, and the patient was treated with radiation therapy. However, he died 2 months later from multiple lung metastases. We thought that the metastases were from the UPS, because his prostatic carcinoma had not progressed. According to published literatures, the UPS rarely accompanies with surface ulceration. On the other hand, SCC commonly occurs on sun-exposed sites of the head and neck with surface changes including scaling, ulceration, crusting, and the presence of a cutaneous horn. In our case, it was very difficult to distinguish UPS from SCSCC by clinical examination and conventional histopathological staining (e.g. hematoxylin and eosin). Therefore, the immunohistochemistry was required to obtain the final diagnosis.
\end{abstract}

Keywords: Undifferentiated Pleomorphic Sarcoma; Ulcer; Spindle Cell Squamous Cell Carcinoma

\section{Introduction}

Undifferentiated pleomorphic sarcoma (UPS), also called malignant fibrous histiocytoma (MFH), belongs to the soft tissue tumors and is more likely in elder patients. UPS currently accounts for approximately $18 \%$ of cutaneous soft tissue sarcoma except Kaposi sarcoma [1].

${ }^{*}$ Corresponding author.
The most common sites are the extremities and head and neck [1]. UPS is rapidly enlarging, and easily invades subcutaneous tissue and muscle without overlying skin changes [1]. UPS tumors, which are confined to subcutaneous tissue, have been reported to be less than $10 \%$. On the other hand, the involvement of the epidermis, occasionally with ulceration, is rare [2]. Here, we describe a case of UPS of the cheek with surface ulceration. 


\section{The Case Report}

An 83-year-old man presented with a 1-year history of a red nodule on his left cheek. He had been treated with antibiotics at another dermatological clinic; however, the size of the nodule gradually increased. He also suffered from severe Alzheimer's dementia and prostatic carcinoma. He had no family history of skin disease. Clinical examination revealed a $55 \times 45 \mathrm{~mm}$ red, hard mass in the center of his left cheek with a large crater-shaped ulcer involving the subcutaneous tissue. The bottom of the ulcer contained necrotic tissue. No cervical lymph nodes were palpable (Figure 1). A biopsy revealed a highgrade, pleomorphic spindle cell neoplasm and frequent abnormal mitotic figures within the dermis. We temporarily diagnosed suspected a soft tissue sarcoma, but we could not rule out spindle cell squamous cell carcinoma (SCSCC).

The patient underwent tumor resection, which involved wide local excision, parotidectomy, and skin grafting. The tumor had reached the muscle layer of his left cheek with invasion and ulceration of the overlying skin. Histopathological examination of the totally resected tumor revealed a neoplasm of mesenchymal origin, rich in cells with morphology and immunohistochemical characteristics compatible with UPS (Figure 2). Malignant cells were not found in the epidermis. Immunohistochemical analyses were positive for vimentin, $\alpha$-antitrypsin, $\alpha$-smooth muscle actin ( $\alpha$-SMA), and CD10, and negative for cytokeratins (CAM5.2 and AE1/AE3), lysozyme, epithelial membrane antigen (EMA), CD34, S100, desmin, and caldesmon. The CD10 usually appears

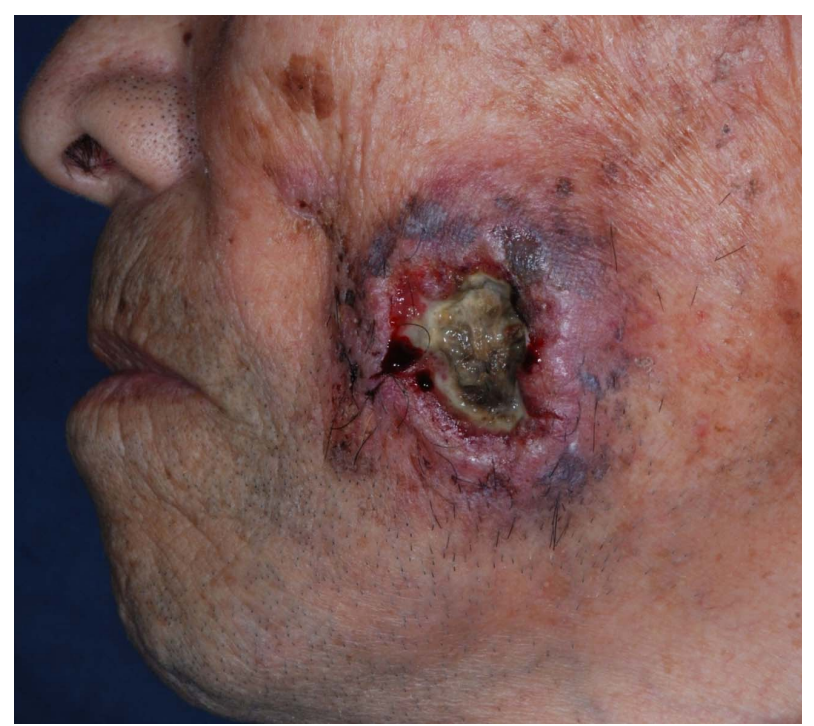

Figure 1. The mass measured $55 \times 45 \mathrm{~mm}$ on the left cheek, it was firm to red color and hard in consistency and fixed to underlying muscles. Overlying skin was ulcerated, inflamed with irregular margins.

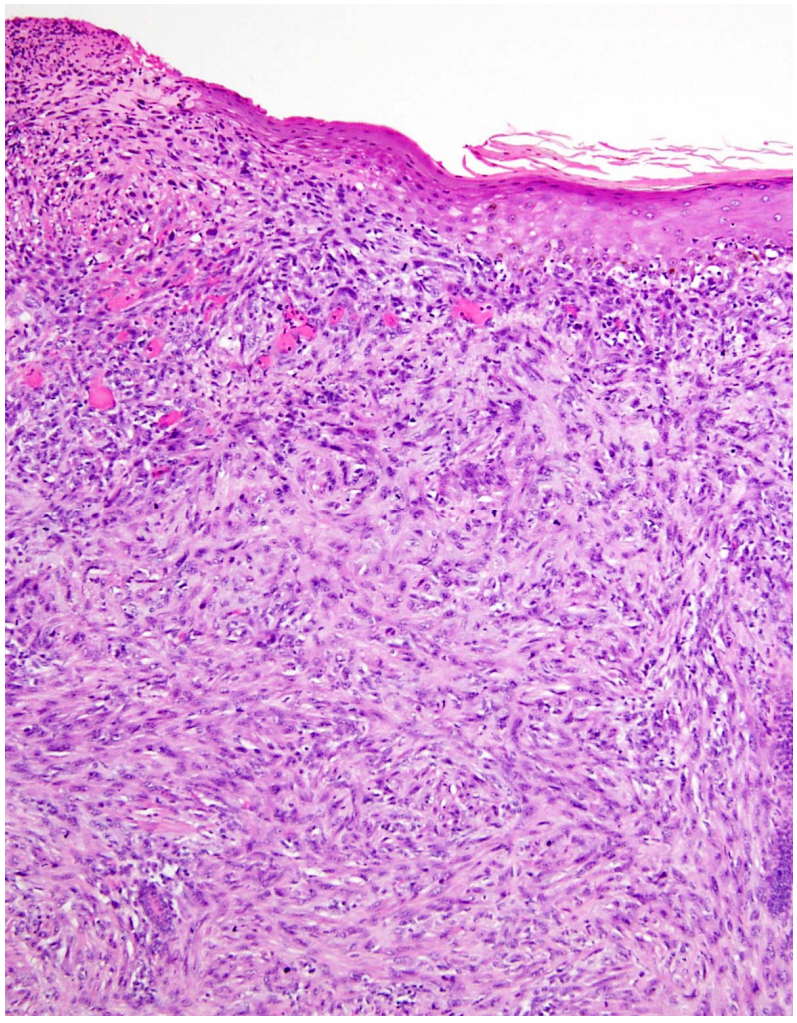

Figure 2. In total resected sample of the tumor, tumor cells were not found in the epidermis. There were tumors cell from the epidermal right under to muscle. The tumor was composed of atypical fibroblastic spindle cells $(\mathrm{H} \& \mathrm{E}, \times 100)$.

in UPS and also atypical fibroxanthoma (AFX) etc. [3,4]. Then we distinguished UPS from AFX by two reasons following, 1) the tumor was larger than $2 \mathrm{~cm}, 2$ ) the tumor cells extensively involved the subcutaneous tissue and penetrated the muscle [5]. Then, we finally diagnosed UPS.

Two months after total resection, the tumor recurred, and the patient was treated with radiation therapy (total of 68 Gray). However, he died 2 months later from multiple lung metastases. We thought that the metastases were from the UPS, because his prostatic carcinoma had not progressed.

\section{Discussion}

According to published literatures, the UPS rarely accompanies with surface ulceration [6]. On the other hand, SCC commonly occurs on sun-exposed sites of the head and neck with surface changes including scaling, ulceration, crusting, and the presence of a cutaneous horn [7].

\section{Conclusion}

In our case, it was very difficult to distinguish UPS from SCSCC by clinical examination and conventional histo- 
pathological staining (e.g. hematoxylin and eosin). Therefore, the immunohistochemistry was required to obtain the final diagnosis.

\section{REFERENCES}

[1] S. T. Hollmig, R. Sachdev, C. J. Cockerell, W. Posten, M. Chiang and J. Kim, "Spindle Cell Neoplasms Encountered in Dermatologic Surgery a Review,” Dermatologic Surgery, Vol. 38, No. 6, 2012, pp. 825-850. http://dx.doi.org/10.1111/j.1524-4725.2012.02296.x

[2] E. E. David, E. Rosalie, L. J. Bernett and F. M. George, "Lever's Histopathology of the Skin," 9th Edition, Lippincott Williams \& Wilkins, Philadelphia, 2005, p. 989.

[3] D. Kemal, C. Ganime and O. Turhan, “Anti-CD10 (56C6) Expression in Soft Tissue Sarcomas,” Pathology, Research and Practice, Vol. 208, No. 5, 2012, pp. 281-285. http://dx.doi.org/10.1016/j.prp.2012.02.002

[4] C. N. Wieland, R. Dyck, R. H. Weenig and N. I. Comfere, "The Role of CD10 in Distinguishing Atypical Fibroxan- thoma from Sarcomatoid (Spindle Cell) Squamous Cell Carcinoma," Journal of Cutaneous Pathology, Vol. 38, No. 11, 2011, pp. 884-888. http://dx.doi.org/10.1111/j.1600-0560.2011.01768.x

[5] S. Shuji, W. Shoichi, K. Hiroshi, I. Hiroshi, H. Hideo and A. Morita, "A Case of Cutaneous Malignant Fibrous Histiocytoma with Multiple Organ Metastases," Kaohsiung Journal of Medical Sciences, Vol. 29, No. 2, 2013, pp. 111-115. http://dx.doi.org/10.1016/j.kjms.2012.08.019

[6] A. Prerna, W. Rachna, K. Nita, J. Shyama and N. S. Hadke, "Secondary Malignant Fibrous Histiocytoma of Thigh with Surface Ulceration Following Radiotherapy for Carcinoma Cervix," Archives of Gynecology and Obstetrics, Vol. 283, Suppl. 1, 2011, pp. S79-S82. http://dx.doi.org/10.1007/s00404-010-1699-3

[7] H. M. Gloster and K. Neal, "Skin Cancer in Skin of Color," Journal of the American Academy of Dermatology, Vol. 55, No. 5, 2006, pp. 741-760.

http://dx.doi.org/10.1016/j.jaad.2005.08.063 